Publications of the Astronomical Society of the Pacific

104: 1205-1215, 1992 December

\title{
Photometry of RR Lyrae Variables in the Globular Clusters M5 and M92
}

\author{
J. G. COHEN \\ Palomar Observatory, Mail Code 105-24, California Institute of Technology, Pasadena, California 91125 \\ Electronic mail: jlc@ deimos.caltech.edu \\ K. MATTHEWS \\ Palomar Observatory, Mail Code 320-47, California Institute of Technology, Pasadena, California 91125 \\ Electronic mail: kym@ tacos.caltech.edu \\ Received 1992 March 10; accepted 1992 August 25
}

\begin{abstract}
Visual photometry at $V$ and $i$ has been obtained for five RR Lyrae variables in the extremely metal-poor globular cluster M92 and eight in the cluster M5, of intermediate metallicity, for use in a Baade-Wesselink analysis. The periods of these variables have been updated by combining observations of the current epoch with older data from the literature. Mean magnitudes are derived from the light curves. Infrared photometry at $J$ and $K$ has also been obtained for two RR Lyrae variables in M92 and for four variables in the cluster M5. The data were obtained with a $58 \times 62$ pixel InSb array. The reduction and analysis techniques are discussed. Preliminary distance determinations using the $\left\langle M_{V}\right\rangle-[\mathrm{Fe} / \mathrm{H}]$ relationship from the literature were derived as $(m-M)_{0}=14.48 \pm 0.04$ for M92 and $14.25 \pm 0.05$ for M5, while preliminary distance determinations using the $\left\langle M_{K}\right\rangle-\log (P)$ relationship were derived as $0.15 \mathrm{mag}$ smaller.
\end{abstract}

\section{INTRODUCTION}

We present visual photometry for five RR Lyrae variables in the extremely metal-poor globular cluster M92 and eight in the intermediate-metallicity cluster M5. Infrared photometry is presented for two RR Lyraes in M92 and four in the globular cluster M5. These data form the basis for a Baade-Wesselink analysis of the distance to these globular-cluster variables in Cohen (1992). The large difference in metallicity between M92 and M5 will be important in efforts to understand the dependence of mean absolute $V$ magnitude of the variables on metallicity, and to see if the globular-cluster stars obey the same relationships that prevail among the field RR Lyraes.

In addition, the globular-cluster RR Lyrae variables are of interest in their own right, as examples of high-precision light curves, for studying the bump in the light curve near phase $\approx 0.7$, for period-shift analyses (see, e.g., Sandage 1990), and for studying possible period changes.

The advantages of infrared photometry in carrying out a Baade-Wesselink method have been amply discussed by Jameson et al. (1987) and by Jones et al. (1988). To summarize briefly, in addition to reducing the impact of any interstellar reddening, the use of $K$, a color far out on the Rayleigh-Jeans tail, reduces the sensitivity of the emitted flux to gravity, metallicity, and the influence of shock waves. In addition, the $(V-K)$ color offers a very broad wavelength baseline so that $T_{\text {eff }}$ can be determined very reliably.

In this paper, since infrared photometry of variable stars with infrared array detectors is still relatively new, we present the data, discuss the data analysis and some of the sources of error, and give the derived mean light curve parameters.
We have two stars, one in each of the clusters, in common with the recent analysis of Storm et al. (1992), who have also embarked on a similar program. In the visual, we have four variables in common with the study of Carney et al. (1992) of M92, and five in common with Storm et al.'s (1991) analysis of M5.

\section{OBSERVATIONS}

\subsection{Instrumental Parameters}

The visual photometry was obtained with the 60 -in. telescope at Palomar Mountain on the six nights of 1989 June 16-17 and 1989 June 20-23. Five of these were simultaneous with the infrared photometry obtained on the 200-in. Hale telescope described later. The visual data were obtained with a direct imaging CCD at the Cassegrain focus using interference filters designed to match the Johnson $V$ filter and the $i$ filter of the Thuan-Gunn (1976) system, which has $\lambda_{\text {eff }} \approx 8100$ A. A $800 \times 800$ pixel TI CCD was used, binned to $400 \times 400$ pixels, to give a scale of 0.48 arcsec pixel $^{-1}$. The output was digitized to two electrons $\mathrm{DN}^{-1}$, with a ten-electron readout noise. The seeing was good; 1-1.5 arcsec every night except the last, when it was 2 arcsec. Four of the nights were photometric; two nights were cirrusy, and there was one where several hours were lost to smoke from a forest fire. Although not all the nights were photometric, local standards in each field could be used to tie the magnitudes of each frame together.

There were two CCD fields studied in M5, with the second being closer to the center of the cluster and hence more crowded, and one in M92. Exposures of $30 \mathrm{~s}$ in length for the M5 fields, and $40 \mathrm{~s}$ for the M92 field were used. 
TABLE 1

Ephemerides of M5 and M92 RR Lyrae Variables

\begin{tabular}{lcccc}
\hline \hline \multicolumn{1}{c}{ Name } & Period & $\begin{array}{c}\text { Zero phase } \\
\text { (Julian Days) }\end{array}$ & $\begin{array}{c}\text { Beta } \\
\text { (days/10 } 0^{6} \text { yr) }\end{array}$ & Notes \\
\hline Field 1 of M5 & & & & \\
M5-V32 & 0.45778654 & $2,446,536.587$ & & \\
M5-V8 & 0.5462390 & $2,447,693.443$ & 0.16 & RRc \\
M5-V31 & $\mathbf{0 . 3 0 0 5 8 2 9 4}$ & $2,446,536.731$ & & \\
M5-V28 & 0.5439272 & $2,446,536.666$ & & \\
M5-V18 & 0.464233 & $2,447,693.519$ & & \\
Field 2 of M5 & & & & \\
M5-V59 & 0.5420257 & $2,446,536.791$ & & \\
M5-V12 & 0.467704 & $2,447.693 .133$ & -0.21 & \\
M5-V79 & 0.33313838 & $2,446,536.814$ & & \\
M92 Field & & & & \\
M92-V1 & 0.7027955 & $2,447,693.233$ & & \\
M92-V2 & 0.6438847 & $2,427,693.458$ & & \\
M92-V6 & 0.599999 & $2,447,693.510$ & & \\
M92-V10 & 0.3772990 & $2,447,693.492$ & & \\
M92-V5 & 0.6196639 & $2,447,693.231$ & & \\
\hline \hline
\end{tabular}

TABLE 2

Light curves of M92 Variables at $V$

\begin{tabular}{lccccc}
\hline \hline Phase & M92-V1 & M92-V2 & M92-V5 & M92-V6 & M92-V10 \\
\hline 0.00 & 14.60 & 14.55 & 14.58 & 14.41 & 14.95 \\
0.02 & 14.64 & 14.56 & 14.60 & 14.47 & 14.95 \\
0.04 & 14.69 & 14.62 & 14.64 & 14.53 & 14.96 \\
0.06 & 14.72 & 14.67 & 14.68 & 14.57 & 14.96 \\
0.08 & 14.77 & 14.73 & 14.75 & 14.63 & 14.97 \\
0.10 & 14.81 & 14.78 & 14.82 & 14.67 & 14.99 \\
0.12 & 14.84 & 14.83 & 14.87 & 14.73 & 15.00 \\
0.14 & 14.86 & 14.88 & 14.90 & 14.77 & 15.02 \\
0.16 & 14.87 & 14.94 & 14.96 & 14.82 & 15.03 \\
0.18 & 14.88 & 14.96 & 14.99 & 14.86 & 15.06 \\
0.20 & 14.89 & 14.98 & 15.01 & 14.89 & 15.09 \\
0.22 & 14.91 & 15.02 & 15.07 & 14.93 & 15.10 \\
0.24 & 14.93 & 15.04 & 15.10 & 14.97 & 15.13 \\
0.26 & 14.96 & 15.06 & 15.12 & 15.02 & 15.15 \\
0.28 & 15.00 & 15.09 & 15.13 & 15.04 & 15.17 \\
0.30 & 15.05 & 15.12 & 15.14 & 15.07 & 15.19 \\
0.32 & 15.09 & 15.15 & 15.15 & 15.09 & 15.21 \\
0.34 & 15.11 & 15.18 & 15.16 & 15.14 & 15.24 \\
0.36 & 15.15 & 15.21 & 15.19 & 15.18 & 15.28 \\
0.38 & 15.18 & 15.21 & 15.23 & 15.22 & 15.31 \\
0.40 & 15.20 & 15.22 & 15.24 & 15.24 & 15.32 \\
0.42 & 15.22 & 15.25 & 15.29 & 15.27 & 15.36 \\
0.44 & 15.23 & 15.26 & 15.32 & 15.30 & 15.37 \\
0.46 & 15.24 & 15.30 & 15.36 & 15.32 & 15.38 \\
0.48 & 15.26 & 15.32 & 15.37 & 15.34 & 15.39 \\
0.50 & 15.27 & 15.36 & 15.40 & 15.35 & 15.40 \\
0.52 & 15.28 & 15.39 & 15.38 & 15.35 & 15.41 \\
0.54 & 15.29 & 15.40 & 15.39 & 15.36 & 15.44 \\
0.56 & 15.30 & 15.41 & 15.41 & 15.36 & 15.45 \\
0.58 & 15.32 & 15.42 & 15.41 & 15.36 & 15.44 \\
0.60 & 15.31 & 15.42 & 15.41 & 15.36 & 15.43 \\
0.62 & 15.31 & 15.41 & 15.42 & 15.39 & 15.44 \\
0.64 & 15.31 & 15.41 & 15.41 & 15.42 & 15.43 \\
0.66 & 15.31 & 15.41 & 15.42 & 15.45 & 15.43 \\
0.68 & 15.31 & 15.41 & 15.42 & 15.47 & 15.42 \\
0.70 & 15.33 & 15.38 & 15.42 & 15.46 & 15.40 \\
0.72 & 15.34 & 15.37 & 15.40 & 15.47 & 15.38 \\
0.74 & 15.35 & 15.33 & 15.40 & 15.46 & 15.35 \\
0.76 & 15.36 & 15.35 & 15.43 & 15.45 & 15.30 \\
0.78 & 15.38 & 15.37 & 15.46 & 15.42 & 15.25 \\
0.80 & 15.41 & 15.41 & 15.50 & 15.40 & 15.20 \\
0.82 & 15.43 & 15.43 & 15.48 & 15.41 & 15.15 \\
0.84 & 15.45 & 15.45 & 15.51 & 15.42 & 15.10 \\
0.86 & 15.45 & 15.46 & 15.53 & 15.44 & 15.07 \\
0.88 & 15.40 & 15.43 & 15.55 & 15.45 & 15.04 \\
0.90 & 15.30 & 15.40 & 15.48 & 15.40 & 15.02 \\
0.92 & 15.10 & 15.27 & 15.29 & 15.24 & 15.00 \\
0.94 & 14.88 & 15.05 & 15.01 & 14.89 & 14.97 \\
0.96 & 14.77 & 14.87 & 14.78 & 14.72 & 14.96 \\
0.98 & 14.66 & 14.65 & 14.63 & 14.47 & 14.95 \\
\hline & & & & &
\end{tabular}

TABLE 3

Light curves of M92 Variables at $i$

\begin{tabular}{|c|c|c|c|c|c|}
\hline Phase & M92-V1 & M92-V2 & M92-V 5 & M92-V6 & M92-V10 \\
\hline 0.00 & 14.91 & 14.90 & 14.91 & 14.85 & 15.24 \\
\hline 0.02 & 14.93 & 14.91 & 14.92 & 14.88 & 15.25 \\
\hline 0.04 & 14.95 & 14.94 & 14.95 & 14.92 & 15.26 \\
\hline 0.06 & 14.98 & 14.97 & 14.99 & 14.96 & 15.26 \\
\hline 0.08 & 15.00 & 15.01 & 15.05 & 14.97 & 15.27 \\
\hline 0.10 & 15.02 & 15.04 & 15.10 & 14.99 & 15.28 \\
\hline 0.12 & 15.03 & 15.07 & 15.13 & 15.02 & 15.30 \\
\hline 0.14 & 15.03 & 15.10 & 15.16 & 15.04 & 15.31 \\
\hline 0.16 & 15.04 & 15.14 & 15.18 & 15.06 & 15.33 \\
\hline 0.18 & 15.05 & 15.17 & 15.20 & 15.08 & 15.34 \\
\hline 0.20 & 15.07 & 15.18 & 15.21 & 15.10 & 15.35 \\
\hline 0.22 & 15.08 & 15.19 & 15.23 & 15.11 & 15.35 \\
\hline 0.24 & 15.09 & 15.20 & 15.25 & 15.14 & 15.36 \\
\hline 0.26 & 15.10 & 15.20 & 15.26 & 15.16 & 15.37 \\
\hline 0.28 & 15.11 & 15.21 & 15.26 & 15.18 & 15.38 \\
\hline 0.30 & 15.13 & 15.22 & 15.25 & 15.19 & 15.39 \\
\hline 0.32 & 15.15 & 15.23 & 15.24 & 15.21 & 15.42 \\
\hline 0.34 & 15.18 & 15.23 & 15.24 & 15.24 & 15.43 \\
\hline 0.36 & 15.21 & 15.23 & 15.25 & 15.26 & 15.44 \\
\hline 0.38 & 15.23 & 15.24 & 15.28 & 15.29 & 15.46 \\
\hline 0.40 & 15.25 & 15.24 & 15.30 & 15.31 & 15.47 \\
\hline 0.42 & 15.26 & 15.24 & 15.34 & 15.32 & 15.49 \\
\hline 0.44 & 15.27 & 15.25 & 15.36 & 15.33 & 15.51 \\
\hline 0.46 & 15.27 & 15.28 & 15.39 & 15.34 & 15.52 \\
\hline 0.48 & 15.27 & 15.29 & 15.41 & 15.36 & 15.53 \\
\hline 0.50 & 15.28 & 15.31 & 15.42 & 15.38 & 15.54 \\
\hline 0.52 & 15.28 & 15.35 & 15.42 & 15.40 & 15.55 \\
\hline 0.54 & 15.30 & 15.38 & 15.43 & 15.41 & 15.56 \\
\hline 0.56 & 15.31 & 15.39 & 15.44 & 15.41 & 15.56 \\
\hline 0.58 & 15.33 & 15.40 & 15.44 & 15.42 & 15.55 \\
\hline 0.60 & 15.32 & 15.40 & 15.45 & 15.42 & 15.55 \\
\hline 0.62 & 15.32 & 15.40 & 15.45 & 15.43 & 15.55 \\
\hline 0.64 & 15.31 & 15.40 & 15.45 & 15.44 & 15.56 \\
\hline 0.66 & 15.32 & 15.39 & 15.46 & 15.46 & 15.55 \\
\hline 0.68 & 15.33 & 15.35 & 15.47 & 15.49 & 15.54 \\
\hline 0.70 & 15.35 & 15.36 & 15.47 & 15.50 & 15.54 \\
\hline 0.72 & 15.36 & 15.34 & 15.47 & 15.50 & 15.53 \\
\hline 0.74 & 15.37 & 15.33 & 15.47 & 15.51 & 15.52 \\
\hline 0.76 & 15.38 & 15.33 & 15.50 & 15.51 & 15.50 \\
\hline 0.78 & 15.40 & 15.38 & 15.53 & 15.50 & 15.47 \\
\hline 0.80 & 15.43 & 15.41 & 15.56 & 15.48 & 15.45 \\
\hline 0.82 & 15.46 & 15.44 & 15.58 & 15.49 & 15.40 \\
\hline 0.84 & 15.47 & 15.47 & 15.60 & 15.50 & 15.37 \\
\hline 0.86 & 15.49 & 15.51 & 15.61 & 15.50 & 15.34 \\
\hline 0.88 & 15.46 & 15.50 & 15.62 & 15.50 & 15.32 \\
\hline 0.90 & 15.43 & 15.48 & 15.55 & 15.51 & 15.30 \\
\hline 0.92 & 15.22 & 15.40 & 15.40 & 15.43 & 15.28 \\
\hline 0.94 & 15.09 & 15.23 & 15.23 & 15.25 & 15.26 \\
\hline 0.96 & 15.06 & 15.13 & 15.12 & 15.03 & 15.25 \\
\hline 0.98 & 14.96 & 14.96 & 14.99 & 14.90 & 15.24 \\
\hline
\end{tabular}

This gave a net count rate for a M92 RR Lyrae of 35,000 $\mathrm{DN}$ at $V$, with the sky contributing about $250 \mathrm{DN}$ pixel $^{-1}$ at $i$, somewhat less than this at $V$. Over the 6 nights of observation there are about $350 V$ frames and about $300 i$ frames of M92, and about $100 V$ frames and $80 i$ frames for each of the 2 fields covered in M5.

\subsection{Data Analysis of the Visual Photometry}

First all the frames were flattened using standard techniques with dome flat fields taken before and after each night's observing. All the frames of a given field were then shifted by an integer number of pixels in $x$ and $y$ so that the fields are aligned to overlie each other. Since the cluster fields are rather crowded, they were analyzed using the profile fitting routine PEAK of DAOPHOT (Stetson 1987). (The crowding was not so bad that the full capability of DAOPHOT's multiple overlapping stellar profile fitting routine NSTAR was felt necessary.) Since these frames were analyzed in as automatic a manner as possible, there may 
be rare cases of overlapping defects, cosmic rays, and other such that have not been weeded out manually.

The variables on each of the frames were analyzed differentially relative to a set of from 10 to 16 uncrowded bright stars on each field. The differential comparisons yielded the number of stars matched up (not the full complement, because often some stars shifted off the edge of the frame during the alignment process), the mean difference in the PEAK magnitudes between the differential standards on the frame and on the adopted calibration list, and the rms dispersion about that mean. When the frames are correctly aligned relative to each other, $\sigma=0.02$ to 0.04 mag for 12 stars. This procedure yielded magnitudes of the RR Lyrae variables relative to the differential standards. In the case of a few M92 variables near the edges of the frames, perfect alignment of the frames was sometimes not achieved, and the magnitudes show a larger than expected scatter.

The magnitudes are calibrated to those of the photometric standards observed on the night of 1989 June 23. Six standard stars from the list of Thuan and Gunn were observed at $V$, and there are nine standard star observations at $i$. Visual inspection of the field of each star on the list of differential standards for nearby neighbors produced a shorter list of completely uncrowded bright differential standard stars. In the numbering system of Arp (1962), they are M5 IV-26, IV-88, IV-80, IV-85, IV-87, and IV-86 for the first M5 field, and III-2, III-16, III-15, II-16, III-6, III-26, III-12, and III-9 for the second M5 field. For M92, the standards were I-28, II-104, XII-34, and XII-45 in the numbering system of Sandage and Walker (1966). Large aperture photometry was carried out using Figaro for each of these to define the aperture growth curve and hence the aperture correction, and the combination of these, the Palomar standard extinction coefficients, and their DAOPHOT PEAK magnitudes defined the relative calibration to the bright photometric standards. The visual photometry of the M5 and M92 RR Lyraes is given in Tables A1 to A6.

\subsection{Comparison with Previous Visual Photometry}

For M92, much of the published photometry is analysis of very deep CCD studies of small fields, and is not relevant here. Comparison of the four standard stars between our measurements and those of Sandage and Walker (1966) gives a small systematic difference between Sandage and Walker's magnitudes and the present ones of $\delta(V)=-0.03 \mathrm{mag}$, with $\sigma=0.09 \mathrm{mag}$. Comparison over the full set of our differential standards yields a mean difference of $-0.16 \mathrm{mag}$ with $\sigma=0.08 \mathrm{mag}$. It appears that Sandage and Walker may have had a magnitude dependent scale error, larger at fainter magnitudes. This effect was already noted by Buonanno et al. (1983). Our agreement with the photographic study of Buonanno et al. (1983) is gratifying; for our nine differential standard stars there is a mean difference of $0.00 \mathrm{mag}$ with $\sigma=0.04 \mathrm{mag}$.

For M5, there is a photographic study by Buonanno et al. (1981) using Arp's (1962) photoelectric calibration. For six differential standard stars in field 1, the mean dif- ference is $0.03 \pm 0.02$, while for the more crowded field 2 , the agreement is worse, for eight stars it is $0.09 \pm 0.04$. In both cases our $V$ magnitudes are systematically brighter. Richer and Fahlman (1987), in a very deep colormagnitude study of M5, found their $V$ magnitudes to be systematically $0.06 \pm 0.07$ brighter than Buonanno et al.'s. Thus comparison with previous photometry of M5 indicates that agreement with the most reliable previous photometry is good, and our magnitude scale is correct.

\section{RESULTS}

\subsection{RR Lyrae Periods and Phases}

Five RR Lyrae variables in M92 were studied. Their positions relative to the cluster center are given in Sawyer Hogg (1973). The initial guesses at period and phase were taken from Kukharkin and Kukharkina (1980), or, for M92 V6, which was not included in that work, from Sawyer Hogg (1973). The periods needed updating in most cases, as manifested by small shifts in phase. The periods were corrected by using the published period as an initial guess, and forcing an integral number of cycles to have elapsed between the zero phase determined from the 1989 June data and the previous epoch of observation. The adopted periods, rate of change of the period $(d P / d t)$, and phase zero points are listed in Table 1.

Kukharkin and Kukharkina (1980) gave an extensive discussion of the variation of period of these RR Lyrae variables in M92 with epoch of observation. They found many abrupt period changes, but no firm case of a secularly changing period. We find two cases where the period decreased in the recent past, and three where it increased. As might be expected, there is good agreement between our new periods and those calculated by Carney et al. (1992).

For M5, we repeated the same two fields used by Cohen and Gordon (1987), which cover 8 RR Lyraes. Their phases and periods were used as initial guesses. They were adequate for all the M5 variables except M5 V8, V12, and V18, where apparently period changes have occurred since 1986.

Coutts and Sawyer Hogg (1969) have given an extensive discussion of period shifts among the M5 RR Lyraes with data reaching back to 1889 . They claim that V18 is too irregular to derive a period shift. We agree; the period changed by so much that the phase was off between 1986 and 1989 by 0.32 cycles. Coutts and Sawyer Hogg believe that the period of M5-V8 is increasing with time. We concur. Similarly, they believe M5-V12 has a decreasing period. Again we concur. But the period differences we derive over the three-year time baseline are about a factor of 2 larger than would be predicted by their coefficients for the rate of change of the period $(d P / d t)$ for these two stars.

\subsection{Mean Magnitudes}

As the visual photometry is very extensive and of high accuracy, we give the mean $V$ and $i$ light curves for the RR Lyraes in M92 in Tables 2 and 3, and the mean light curves for the RR Lyraes in M5 are given in Tables 4 and 5. 
TABLE 4

Light curves of M5 Variables at $V$

\begin{tabular}{|c|c|c|c|c|c|c|c|c|}
\hline Phase & M5.V8 & M5-V12 & M5-V18 & M5-V28 & M5.V31 & M5-V32 & M5-V59 & M5.V79 \\
\hline 0.00 & 14.530 & 14.330 & & 14.560 & 14.880 & 14.290 & & 14.810 \\
\hline 0.02 & 14.590 & 14.410 & & 14.580 & 14.870 & 14.310 & & 14.810 \\
\hline 0.04 & 14.620 & 14.460 & & 14.620 & 14.880 & 14.350 & & 14.810 \\
\hline 0.06 & 14.640 & 14.520 & 14.740 & 14.680 & 14.890 & 14.420 & & 14.830 \\
\hline 0.08 & 14.670 & 14.580 & 14.770 & 14.740 & 14.900 & 14.490 & & 14.860 \\
\hline 0.10 & 14.700 & 14.650 & 14.800 & 14.790 & 14.920 & 14.560 & & 14.870 \\
\hline 0.12 & 14.740 & $\begin{array}{l}14.700 \\
1.770\end{array}$ & 14.840 & 14.840 & 14.930 & 14.620 & & 14.880 \\
\hline 0.14 & 14.810 & 14.770 & 14.910 & 14.860 & 14.940 & 14.680 & & 14.890 \\
\hline 0.16 & 14.920 & 14.840 & 14.980 & 14.890 & 14.960 & 14.750 & & 14.900 \\
\hline 0.18 & 14.960 & 14.900 & 14.990 & 14.940 & 14.980 & 14.800 & & $\begin{array}{l}14.900 \\
\end{array}$ \\
\hline 0.20 & 14.980 & 14.940 & 15.050 & $\begin{array}{l}14.970 \\
15.900\end{array}$ & 15.000 & 14.840 & & $\begin{array}{l}14.910 \\
\end{array}$ \\
\hline 0.22 & $\begin{array}{l}15.030 \\
\end{array}$ & $\begin{array}{l}14.980 \\
1.900\end{array}$ & 15.090 & $\begin{array}{l}15.000 \\
0.030\end{array}$ & 15.010 & 14.910 & & 14.940 \\
\hline 0.24 & $\begin{array}{l}15.070 \\
\end{array}$ & 15.020 & 15.130 & $\begin{array}{l}15.030 \\
15050\end{array}$ & 15.030 & 14.960 & & 14.960 \\
\hline 0.26 & 15.120 & 15.050 & 15.160 & 15.050 & $\begin{array}{l}15.050 \\
15.070\end{array}$ & 15.000 & $\begin{array}{l}14.920 \\
1.920\end{array}$ & \\
\hline 0.28 & 15.160 & 15.086 & 15.180 & 15.080 & $\begin{array}{l}15.070 \\
5\end{array}$ & $\begin{array}{l}15.040 \\
15.08\end{array}$ & $\begin{array}{l}14.920 \\
\end{array}$ & \\
\hline 0.30 & 15.190 & 15.122 & $\begin{array}{l}15.220 \\
15270\end{array}$ & 15.110 & 15.090 & 15.080 & 14.940 & \\
\hline 0.32 & 15.210 & 15.158 & 15.270 & 15.150 & 15.130 & 15.120 & 14.970 & \\
\hline 0.34 & 15.230 & 15.194 & 15.300 & 15.190 & 15.160 & $\begin{array}{l}15.160 \\
\end{array}$ & $\begin{array}{l}15.000 \\
15\end{array}$ & \\
\hline 0.36 & $\begin{array}{l}15.240 \\
\end{array}$ & 15.230 & 15.320 & $\begin{array}{l}15.210 \\
\end{array}$ & $\begin{array}{l}15.180 \\
15.0\end{array}$ & 15.200 & 15.060 & \\
\hline 0.38 & $\begin{array}{l}15.270 \\
\end{array}$ & $\begin{array}{l}15.260 \\
15.90\end{array}$ & 15.340 & $\begin{array}{l}15.240 \\
\end{array}$ & $\begin{array}{l}15.210 \\
15.20\end{array}$ & $\begin{array}{l}15.230 \\
\end{array}$ & 15.120 & \\
\hline 0.40 & $\begin{array}{l}15.300 \\
\end{array}$ & 15.290 & 15.360 & $\begin{array}{l}15.260 \\
\end{array}$ & $\begin{array}{l}15.240 \\
\end{array}$ & 15.250 & 15.150 & \\
\hline 0.42 & $\begin{array}{l}15.330 \\
\end{array}$ & 15.320 & 15.380 & $\begin{array}{l}15.290 \\
\end{array}$ & $\begin{array}{l}15.280 \\
15300\end{array}$ & $\begin{array}{l}15.290 \\
\end{array}$ & 15.160 & \\
\hline 0.44 & $\begin{array}{l}15.360 \\
\end{array}$ & $\begin{array}{l}15.350 \\
15.380\end{array}$ & 15.410 & 15.320 & $\begin{array}{l}15.300 \\
0\end{array}$ & $\begin{array}{l}15.320 \\
\end{array}$ & 15.190 & \\
\hline 0.46 & $\begin{array}{l}15.360 \\
\end{array}$ & 15.380 & 15.400 & 15.330 & $\begin{array}{l}15.320 \\
\end{array}$ & $\begin{array}{l}15.340 \\
\end{array}$ & $\begin{array}{l}15.210 \\
15.90\end{array}$ & \\
\hline 0.48 & $\begin{array}{l}15.370 \\
\end{array}$ & 15.405 & 15.420 & $\begin{array}{l}15.350 \\
15.3\end{array}$ & $\begin{array}{l}15.350 \\
5.310\end{array}$ & $\begin{array}{l}15.360 \\
\end{array}$ & 15.190 & \\
\hline 0.50 & 15.380 & 15.430 & 15.440 & 15.360 & $\begin{array}{l}15.340 \\
\end{array}$ & 15.380 & 15.200 & \\
\hline 0.52 & $\begin{array}{l}15.390 \\
\end{array}$ & 15.455 & 15.460 & $\begin{array}{l}15.360 \\
\end{array}$ & $\begin{array}{l}15.360 \\
15370\end{array}$ & $\begin{array}{l}15.410 \\
\end{array}$ & 15.220 & \\
\hline 0.54 & 15.390 & 15.480 & 15.490 & 15.370 & $\begin{array}{l}15.370 \\
15.370\end{array}$ & $\begin{array}{l}15.450 \\
15.400\end{array}$ & $\begin{array}{l}15.240 \\
15.240\end{array}$ & \\
\hline $\begin{array}{l}0.56 \\
0.58\end{array}$ & $\begin{array}{l}15.400 \\
15.400\end{array}$ & $\begin{array}{l}15.500 \\
15.505\end{array}$ & $\begin{array}{l}15.490 \\
15.480\end{array}$ & $\begin{array}{l}15.380 \\
15.370\end{array}$ & $\begin{array}{l}15.370 \\
15.370\end{array}$ & $\begin{array}{l}15.470 \\
15.480\end{array}$ & $\begin{array}{l}15.240 \\
15.210\end{array}$ & \\
\hline $\begin{array}{l}0.58 \\
0.60\end{array}$ & $\begin{array}{l}15.400 \\
15.410\end{array}$ & $\begin{array}{l}15.505 \\
15.510\end{array}$ & $\begin{array}{l}15.480 \\
15470\end{array}$ & $\begin{array}{l}15.370 \\
15.370\end{array}$ & $\begin{array}{l}15.370 \\
15.360\end{array}$ & $\begin{array}{l}15.480 \\
15.460\end{array}$ & $\begin{array}{l}15.210 \\
15.210\end{array}$ & \\
\hline 0.62 & $\begin{array}{l}15.410 \\
15.400\end{array}$ & $\begin{array}{l}15.510 \\
15.513\end{array}$ & $\begin{array}{l}15.470 \\
15.500\end{array}$ & $\begin{array}{l}15.370 \\
15.380\end{array}$ & $\begin{array}{l}\begin{array}{l}15.360 \\
15.350\end{array} \\
150\end{array}$ & $\begin{array}{l}15.460 \\
15.450\end{array}$ & $\begin{array}{l}\begin{array}{l}15.210 \\
15.220\end{array} \\
\text { a } 210\end{array}$ & \\
\hline 0.64 & 15.410 & 15.515 & 15.530 & 15.390 & 15.310 & 15,440 & 15.230 & \\
\hline 0.66 & 15.420 & 15.520 & 15.500 & 15.390 & 15.310 & 15.420 & 15.240 & 15.090 \\
\hline 0.68 & 15.430 & 15.530 & 15.490 & 15.390 & 15.320 & 15.440 & 15.240 & 15.060 \\
\hline 0.70 & 15.450 & 15.533 & 15.490 & 15.400 & 15.320 & 15.440 & 15.240 & 15.010 \\
\hline 0.72 & 15.480 & 15.536 & 15.540 & 15.400 & 15.310 & 15.450 & 15.250 & 14.980 \\
\hline 0.74 & 15.520 & 15.539 & 15.530 & 15.400 & 15.290 & 15.460 & 15.240 & 14.950 \\
\hline 0.76 & 15.530 & 15.542 & 15.530 & 15.400 & 15.250 & 15.470 & 15.260 & 14.930 \\
\hline 0.78 & 15.540 & 15.545 & 15.500 & 15.400 & 15.200 & 15.460 & 15.290 & 14.900 \\
\hline 0.80 & 15.550 & 15.548 & 15.470 & 15.420 & 15.150 & 15.440 & 15.320 & 14.880 \\
\hline 0.82 & 15.560 & 15.551 & 15.460 & 15.460 & 15.080 & 15.460 & 15.350 & 14.870 \\
\hline 0.84 & 15.570 & 15.554 & 15.420 & 15.460 & 14.990 & 15.490 & 15.350 & 14.850 \\
\hline 0.86 & $\begin{array}{l}15.510 \\
15100\end{array}$ & $\begin{array}{l}15.557 \\
15.515\end{array}$ & $\begin{array}{l}15.340 \\
5.140\end{array}$ & $\begin{array}{l}15.460 \\
\end{array}$ & $\begin{array}{l}14.920 \\
14910\end{array}$ & $\begin{array}{l}15.540 \\
15590\end{array}$ & $\begin{array}{l}15.370 \\
15.390\end{array}$ & $\begin{array}{l}14.840 \\
14830\end{array}$ \\
\hline 0.88 & $\begin{array}{l}15.160 \\
15\end{array}$ & $\begin{array}{l}15.515 \\
15.46\end{array}$ & $\begin{array}{l}15.140 \\
5\end{array}$ & $\begin{array}{l}15.370 \\
15.200\end{array}$ & $\begin{array}{l}14.910 \\
14900\end{array}$ & $\begin{array}{l}15.590 \\
15.560\end{array}$ & $\begin{array}{l}15.390 \\
15.320\end{array}$ & $\begin{array}{l}14.830 \\
14.820\end{array}$ \\
\hline 0.90 & 15.000 & 15.460 & 15.000 & 15.260 & $\begin{array}{l}14.900 \\
14880\end{array}$ & $\begin{array}{l}15.560 \\
15.460\end{array}$ & $\begin{array}{l}15.320 \\
15.180\end{array}$ & $\begin{array}{l}14.820 \\
14.800\end{array}$ \\
\hline 0.92 & 14.920 & 15.340 & 14.930 & 15.100 & $\begin{array}{r}14.880 \\
14.890\end{array}$ & $\begin{array}{l}15.460 \\
15.240\end{array}$ & $\begin{array}{l}15.180 \\
15.010\end{array}$ & $\begin{array}{l}14.800 \\
14.800\end{array}$ \\
\hline 0.94 & 14.800 & 14.950 & & 14.970 & & $\begin{array}{l}15.240 \\
14880\end{array}$ & 15.010 & $\begin{array}{l}14.800 \\
14.800\end{array}$ \\
\hline $\begin{array}{l}0.96 \\
0.98\end{array}$ & $\begin{array}{l}14.690 \\
14.590\end{array}$ & $\begin{array}{l}14.620 \\
14.430\end{array}$ & & $\begin{array}{l}14.870 \\
14670\end{array}$ & $\begin{array}{l}14.880 \\
14.880\end{array}$ & $\begin{array}{l}14.880 \\
14.460\end{array}$ & & $\begin{array}{l}14.800 \\
14.800\end{array}$ \\
\hline
\end{tabular}

Figures 1 and 2 show the $V$ and $i$ light curves, respectively, for the 13 RR Lyrae variables.

Table 6 contains the magnitude mean and intensity mean parameters of the light curve. No reddening corrections have been applied. M5-79 is omitted from Table 6 due to incomplete phase coverage. For the four RR Lyrae variables in M92 in common with Carney et al. (1992), we find a mean difference of $0.03 \pm 0.03$ for the magnitude mean $V$ values. If the intensity mean values are compared, the difference is $0.05 \pm 0.02$. All the M5 stars in common with Carney et al. (1991) are in field 1. The difference of the magnitude means is $0.05 \pm 0.03$, while the difference of the intensity means yields $0.07 \pm 0.03$.

Using the data in Cohen and Gordon to supply mean magnitudes for M5-V79, we find that both $c$-type RR Lyrae variables in M5 are well separated from the remaining variables in a plot of $(V, V-i)$. This also holds for the only M92 c-type RR Lyrae (M92-V10). This shows that $(V-i)$ is a better indicator of $T_{\text {eff }}$ than is $(B-V)$ (see Storm et al. 1991).

\section{INFRARED PHOTOMETRY}

\subsection{Infrared Observations}

Infrared photometry at $J$ and $K$ was obtained using the Cassegrain IR array camera on the 200-in. Hale telescope on the nights of 1989 June 16-17 and 1989 June 21-23, simultaneously with the optical photometry described above obtained on the 60 -in. telescope. Only four of the five
TABLE 5

Light curves of M5 Variables at $i$

\begin{tabular}{|c|c|c|c|c|c|c|c|c|}
\hline Phase & M5.V8 & M5-V12 & M5-V18 & M5-V28 & M5-V31 & M5-V32 & M5-V59 & M5-V79 \\
\hline 0.00 & 14.82 & 14.830 & & 14.890 & 15.170 & 14.720 & & 15.060 \\
\hline 0.02 & 14.85 & 14.860 & & 14.910 & 15.190 & 14.750 & & 15.065 \\
\hline 0.04 & 14.89 & 14.890 & & 14.930 & 15.200 & 14.780 & & 15.070 \\
\hline 0.06 & 14.91 & 14.920 & 15.040 & 14.970 & 15.210 & 14.830 & & 15.060 \\
\hline 0.08 & 14.93 & 14.960 & 15.070 & 15.010 & 15.210 & 14.880 & & 15.070 \\
\hline 0.10 & 14.95 & 15.015 & 15.090 & 15.030 & 15.220 & 14.920 & & 15.080 \\
\hline 0.12 & 14.98 & 15.045 & 15.120 & 15.040 & 15.220 & 14.960 & & 15.090 \\
\hline 0.14 & 15.00 & 15.080 & 15.150 & 15.050 & 15.220 & 14.990 & & 15.090 \\
\hline 0.16 & 15.02 & 15.120 & 15.170 & 15.080 & 15.230 & 15.030 & & 15.100 \\
\hline 0.18 & 15.04 & 15.140 & 15.180 & 15.090 & 15.240 & 15.070 & & 15.110 \\
\hline 0.20 & 15.06 & 15.170 & 15.200 & 15.100 & 15.260 & 15.110 & & 15.110 \\
\hline 0.22 & 15.08 & 15.190 & 15.250 & 15.120 & 15.270 & 15.150 & & \\
\hline 0.24 & 15.11 & 15.210 & 15.280 & 15.140 & 15.280 & 15.170 & 15.020 & \\
\hline 0.26 & 15.13 & 15.230 & 15.260 & 15.150 & 15.290 & 15.200 & 15.040 & \\
\hline 0.28 & 15.17 & 15.250 & 15.280 & 15.170 & 15.300 & 15.220 & 15.050 & \\
\hline 0.30 & 15.18 & 15.270 & 15.300 & 15.180 & 15.310 & 15.250 & 15.060 & \\
\hline 0.32 & 15.20 & 15.292 & 15.330 & 15.190 & 15.320 & 15.270 & 15.080 & \\
\hline 0.34 & 15.22 & 15.314 & 15.340 & 15.200 & 15.350 & 15.290 & 15.090 & \\
\hline 0.36 & 15.24 & 15.336 & 15.360 & 15.210 & 15.390 & 15.310 & 15.110 & \\
\hline 0.38 & 15.25 & 15.358 & 15.380 & 15.230 & 15.410 & 15.330 & 15.130 & \\
\hline 0.40 & 15.27 & 15.380 & 15.400 & 15.240 & 15.420 & 15.360 & 15.150 & \\
\hline 0.42 & 15.28 & 15.394 & 15.420 & 15.260 & 15.440 & 15.390 & 15.190 & \\
\hline 0.44 & 15.29 & 15.408 & 15.420 & 15.280 & 15.460 & 15.380 & 15.200 & \\
\hline 0.46 & 15.31 & 15.422 & 15.420 & 15.300 & 15.470 & 15.400 & 15.220 & \\
\hline 0.48 & 15.32 & 15.436 & 15.430 & 15.310 & 15.490 & 15.410 & 15.220 & \\
\hline 0.50 & 15.33 & 15.450 & 15.450 & 15.330 & 15.490 & 15.420 & 15.230 & \\
\hline 0.52 & 15.35 & 15.470 & 15.490 & 15.340 & 15.500 & 15.440 & 15.240 & \\
\hline 0.54 & 15.36 & 15.500 & 15.500 & 15.340 & 15.510 & 15.450 & 15.260 & \\
\hline 0.56 & 15.37 & 15.520 & 15.510 & 15.350 & 15.490 & 15.480 & 15.260 & \\
\hline 0.58 & 15.36 & 15.550 & 15.470 & 15.360 & 15.480 & 15.510 & 15.250 & \\
\hline 0.60 & 15.36 & 15.555 & 15.500 & 15.360 & 15.490 & 15.520 & 15.250 & \\
\hline 0.62 & 15.37 & 15.560 & 15.500 & 15.370 & 15.490 & 15.510 & 15.260 & \\
\hline 0.64 & 15.38 & 15.570 & 15.500 & 15.370 & 15.490 & 15.500 & 15.260 & \\
\hline 0.66 & 15.38 & 15.580 & 15.500 & 15.370 & 15.490 & 15.470 & 15.260 & 15.220 \\
\hline 0.68 & 15.39 & 15.585 & 15.500 & 15.380 & 15.500 & 15.450 & 15.260 & 15.220 \\
\hline 0.70 & 15.41 & 15.588 & 15.540 & 15.400 & 15.490 & 15.460 & 15.270 & 15.210 \\
\hline 0.72 & 15.41 & 15.591 & 15.540 & 15.410 & 15.500 & 15.480 & 15.280 & 15.190 \\
\hline 0.74 & 15.42 & 15.594 & 15.540 & 15.420 & 15.480 & 15.500 & 15.280 & 15.170 \\
\hline 0.76 & 15.43 & 15.597 & 15.540 & 15.420 & 15.430 & 15.510 & 15.280 & 15.160 \\
\hline 0.78 & 15.45 & 15.600 & 15.540 & 15.420 & 15.420 & 15.510 & 15.330 & 15.160 \\
\hline 0.80 & 15.49 & 15.603 & 15.530 & 15.420 & 15.410 & 15.490 & 15.350 & 15.150 \\
\hline 0.82 & 15.54 & 15.606 & 15.520 & 15.420 & 15.360 & 15.520 & 15.370 & 15.120 \\
\hline 0.84 & 15.56 & 15.609 & 15.450 & 15.420 & 15.310 & 15.520 & 15.410 & 15.100 \\
\hline 0.86 & 15.56 & 15.612 & 15.410 & 15.420 & 15.260 & 15.550 & 15.430 & 15.080 \\
\hline 0.88 & 15.52 & 15.600 & 15.340 & & 15.240 & 15.560 & 15.410 & 15.070 \\
\hline 0.90 & 15.43 & 15.600 & 15.250 & & 15.220 & 15.570 & 15.370 & 15.060 \\
\hline 0.92 & 15.29 & 15.510 & 15.220 & & 15.210 & 15.510 & 15.260 & 15.060 \\
\hline 0.94 & 15.18 & 15.170 & & & 15.210 & 15.320 & & 15.070 \\
\hline 0.96 & 15.07 & 14.990 & & & 15.190 & 15.180 & & 15.060 \\
\hline 0.98 & 14.91 & 14.860 & & & 15.170 & 14.880 & & 15.060 \\
\hline
\end{tabular}

nights were photometric (the fifth suffered from moderate cirrus), and several hours were lost of another due to smoke from a nearby forest fire. Thus local standards in several of the fields had to be be used to tie the magnitudes of each frame of a particular variable together. The visual seeing was between 1.0 and 1.5 arcsec for four of the five nights and about 2.0 arcsec on the last night.

The camera contains a Hughes Santa Barbara Research Center $58 \times 62$ element InSb array, used at the $f / 70$ lowbackground Cassegrain focus. The scale is 0.31 arcsec pixel ${ }^{-1}$. The array has a dark current of 90 $e$ pixel $^{-1} \mathrm{~s}^{-1}$, and a readout noise of $450 e$. It is digitized to $1 \mathrm{DN} / 90 e$. The array has two large cracks that one must avoid placing stellar images on or near, as well as several bad pixels. Although this was one of the earliest nonengineering runs of this camera, the array performed well and was very stable. Many subsequent experiments have shown the system to be stable in both responsivity and linearity.

It was hoped that by using an array, one could overcome two serious problems which arise in infrared photometry of globular-cluster RR Lyrae variables using single element detectors. The first is the crowding in these cluster fields, which makes observations using aperture photometry difficult unless the seeing is extremely good and stable, so that small aperture sizes can be used. The second constraint is the problem of achieving full phase coverage with a limited allocation of nights on a large telescope, some of which might be nonphotometric. It was hoped that the use of an IR array would permit the same procedure of local standards near each star used so suc- 

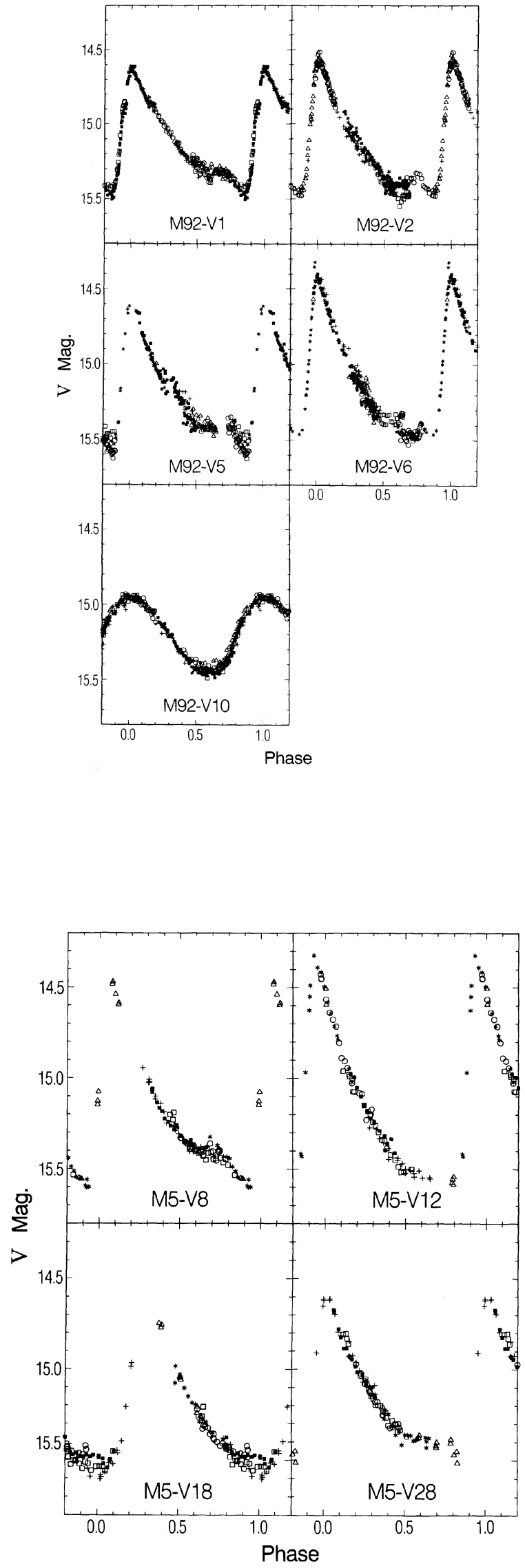

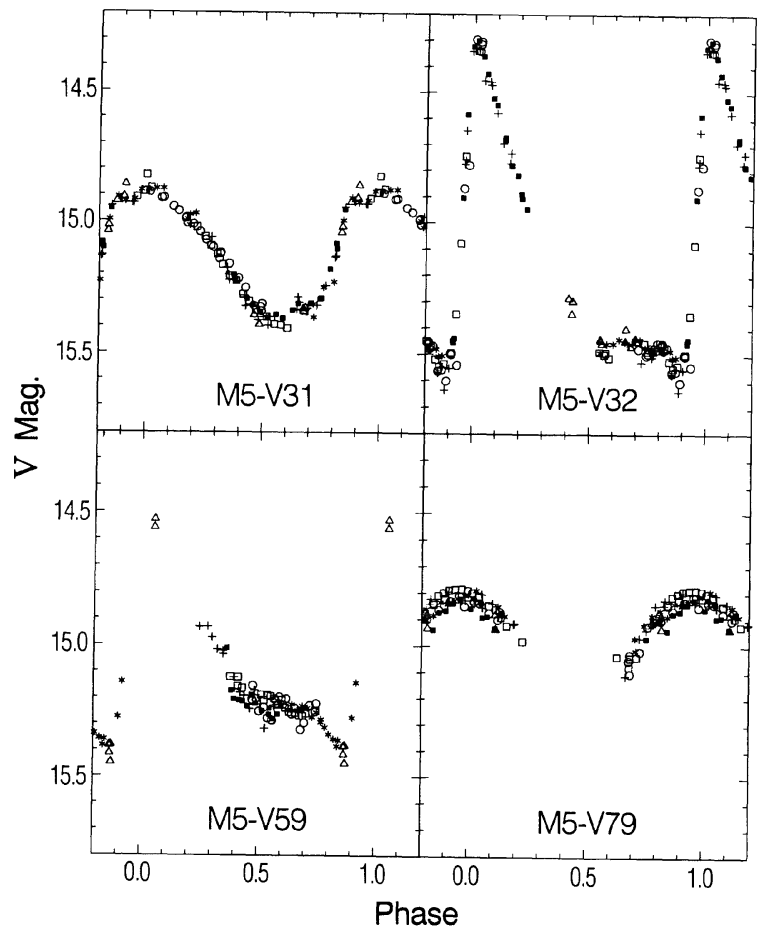

FIG. 1-The $V$ light curves of RR Lyrae variables in M5 and M92 are shown as a function of phase. The different symbols denote different nights of observation. 
COHEN AND MATTHEWS
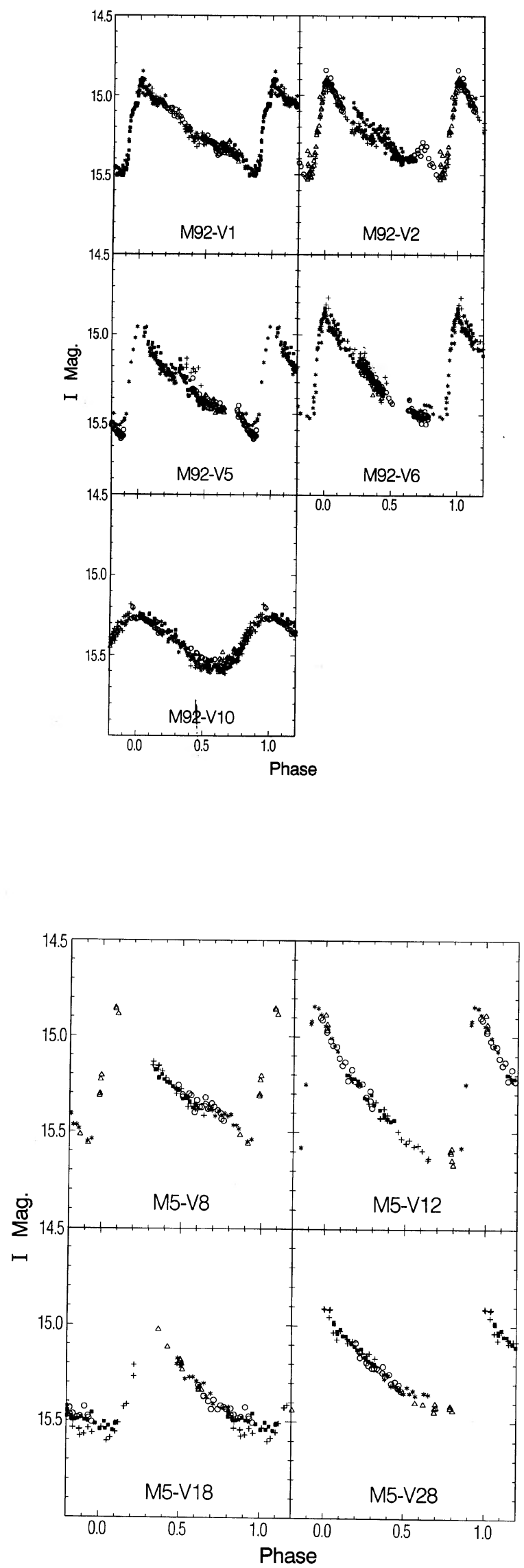

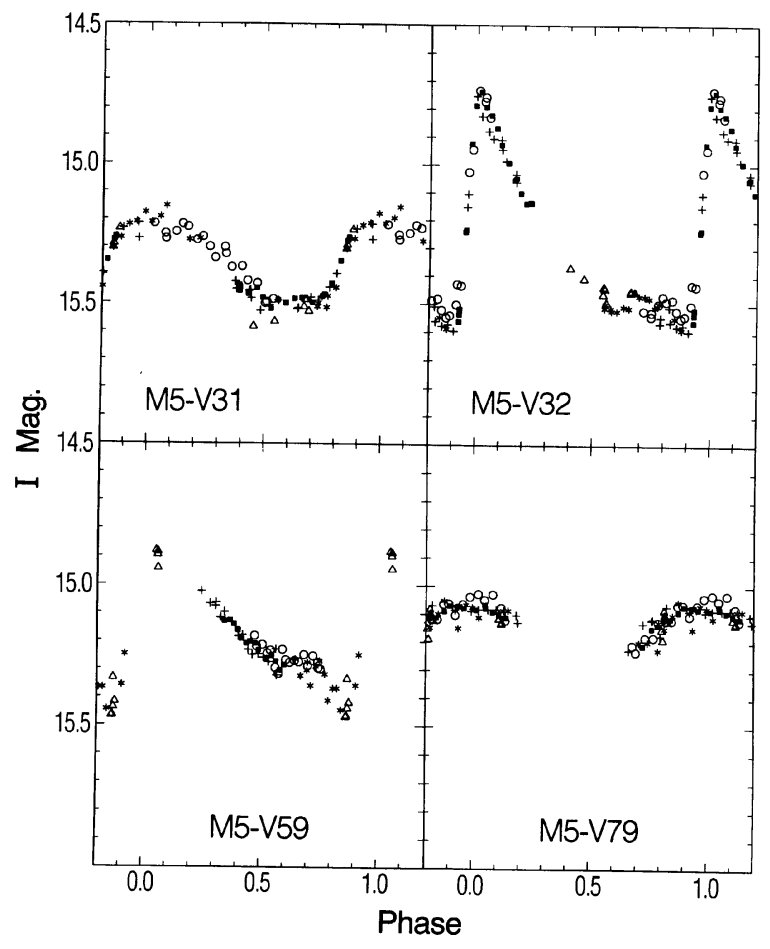

FIG. 2-The $i$ light curves of RR Lyrae variables in M5 and M92 are shown as a function of phase. The different symbols denote different nights of observation. 
TABLE 6

Mean Magnitudes of M92 and M5 RR Lyrae Variables

\begin{tabular}{lcccr}
\hline \hline Star & $\left\langle M_{\mathrm{V}}\right\rangle$ & $\left\langle M_{\mathrm{V}(\text { int })}\right\rangle$ & $\left\langle M_{\mathrm{i}}\right\rangle$ & $\left\langle M_{\mathrm{i} \text { (int) }}\right\rangle$ \\
\hline M5-V8 & 15.166 & 15.146 & 15.236 & 15.228 \\
M5-V12 & 15.184 & 15.154 & 15.340 & 15.327 \\
M5-V18 & 15.196 & 15.148 & 15.319 & 15.312 \\
M5-V28 & 15.158 & 15.143 & 15.225 & 15.220 \\
M5-V31 & 15.118 & 15.111 & 15.344 & 15.347 \\
M5-V32 & 15.143 & 15.113 & 15.290 & 15.277 \\
M5-V59 & 15.032 & 15.016 & 15.135 & 15.128 \\
M92-V1 & 15.119 & 15.107 & 15.213 & 15.208 \\
M92-V2 & 15.164 & 15.149 & 15.254 & 15.249 \\
M92-V5 & 15.192 & 15.176 & 15.320 & 15.313 \\
M92-V6 & 15.129 & 15.108 & 15.274 & 15.265 \\
M92-V10 & 15.202 & 15.195 & 15.412 & 15.409 \\
\hline \hline
\end{tabular}

cessfully at optical wavelengths, so that even if conditions were not ideal, useful data could still be obtained.

While the first advantage of using an array cited above was achieved, the second was only partially realized. In 1989 , infrared arrays were still quite small $(18 \times 19$ arcsec for the array used here), with many localized defects, so one must be quite careful where the object of interest is located if accurate photometry is desired. Hence it was only possible to define local standards around half of the variables.

Since the RR Lyraes in a given cluster are far apart compared to the field size of the IR detector, each RR Lyrae had to be imaged individually with the infrared array camera. Thus, in M92, we obtained IR photometry only for variables M92 V1 and M92 V6. In M5, the same four variables analyzed in detail by Cohen and Gordon (1987) were repeated, M5 V32, M5 V8, M5 V12, and M5 V59.

The fainter standard stars of Elias et al. (1982) were used to reproduce the CIT system. In addition to standard flat fields, dark frames and a sequence of flat fields of varying exposure times were taken at the beginning of each night. The nonlinearity of the array (about $4 \%$ over its useful dynamic range) was measured each night. Integration time for the standard stars was a sum of ten frames, each $0.25 \mathrm{~s}$ long. Eight to ten standard stars were observed each photometric night.

For the globular-cluster RR Lyraes, the exposure time was $20 \mathrm{~s}$, except for the cirrusy night, when 30-s exposures were used. The former gave more than $3000 \mathrm{DN}$ in a typical $K$ image of a variable, and for the $J$ image somewhat more. The sky was typically $500 \mathrm{DN}$ pixel $^{-1}$ in the $J$ exposures, and $3500 \mathrm{DN} \mathrm{pixel}^{-1}$ in the $K$ frames. Saturation is at more than $10,000 \mathrm{DN}_{\text {pixel }}^{-1}$.

A measurement, either of a standard or a program star, consisted of a pair of frames, one through the $J$ filter, and the second through the $K$ filter. After each measurement, a second pair of frames with identical exposure times was taken with the telescope slightly shifted in position by a few arcseconds so as to move the object around on the array. The stars were moved automatically by shifting the autoguider between exposures. Thus each object always fell
TABLE 7

$J$ and $K$ Photometry of RR Lyrae M92-V1

\begin{tabular}{|c|c|c|c|}
\hline $\begin{array}{r}\text { Geocentric } \\
\text { Julian Date } \\
-2,447,000.0\end{array}$ & Phase & $\begin{array}{c}\mathrm{J} \\
(\mathrm{mag})\end{array}$ & $\begin{array}{c}\mathrm{K} \\
(\mathrm{mag})\end{array}$ \\
\hline 693.740 & 0.721 & 14.116 & 13.925 \\
\hline 693.788 & 0.789 & 14.159 & 13.936 \\
\hline 693.826 & 0.843 & 14.180 & 13.987 \\
\hline 693.860 & 0.893 & 14.193 & 14.033 \\
\hline 693.903 & 0.953 & 14.035 & 13.998 \\
\hline 693.915 & 0.970 & 13.923 & 13.858 \\
\hline 693.928 & 0.990 & 13.966 & 13.905 \\
\hline 693.942 & 0.009 & 13.917 & 13.897 \\
\hline 693.958 & 0.031 & 13.856 & 13.837 \\
\hline 693.971 & 0.050 & 13.882 & 13.827 \\
\hline 694.656 & 0.024 & 13.987 & 13.670 \\
\hline 694.688 & 0.071 & 13.915 & 13.661 \\
\hline 694.717 & 0.111 & 13.981 & 13.785 \\
\hline 694.751 & 0.161 & 14.057 & 13.795 \\
\hline 694.788 & 0.213 & 13.939 & 13.701 \\
\hline 694.835 & 0.279 & 13.822 & 13.643 \\
\hline 694.872 & 0.332 & 14.005 & 13.812 \\
\hline 694.886 & 0.352 & 13.965 & 13.834 \\
\hline 694.897 & 0.368 & 14.136 & 13.813 \\
\hline 694.908 & 0.384 & 14.180 & 13.808 \\
\hline 694.919 & 0.400 & 14.162 & 13.857 \\
\hline 694.931 & 0.415 & & 13.791 \\
\hline 694.943 & 0.433 & 13.889 & \\
\hline 694.956 & 0.451 & 14.018 & \\
\hline 694.967 & 0.468 & 13.994 & \\
\hline 694.978 & 0.483 & 14.013 & \\
\hline 694.989 & 0.498 & 14.208 & \\
\hline 698.681 & 0.751 & 14.255 & 13.892 \\
\hline 698.715 & 0.801 & 14.242 & 14.047 \\
\hline 698.758 & 0.861 & 14.214 & 13.964 \\
\hline 698.788 & 0.904 & 14.223 & 13.911 \\
\hline 698.894 & 0.056 & 13.910 & 13.794 \\
\hline 698.913 & 0.081 & 13.992 & 13.831 \\
\hline 698.940 & 0.120 & 13.971 & 13.719 \\
\hline 698.951 & 0.136 & 13.985 & 13.790 \\
\hline 699.677 & 0.169 & 13.912 & 13.722 \\
\hline 699.710 & 0.216 & 13.953 & 13.721 \\
\hline 699.738 & 0.256 & 13.978 & 13.750 \\
\hline 699.766 & 0.296 & 13.962 & 13.709 \\
\hline 699.808 & 0.356 & 14.021 & 13.781 \\
\hline 699.884 & 0.464 & 14.077 & 13.836 \\
\hline 699.893 & 0.476 & 14.035 & 13.796 \\
\hline 699.912 & 0.503 & 14.134 & 13.780 \\
\hline 699.958 & 0.568 & 14.152 & 13.859 \\
\hline 699.979 & 0.598 & 14.180 & 13.922 \\
\hline 700.683 & 0.601 & & 13.859 \\
\hline 700.714 & 0.644 & 14.184 & 13.849 \\
\hline 700.751 & 0.697 & 14.157 & 13.769 \\
\hline 700.780 & 0.738 & 14.182 & 13.887 \\
\hline 700.819 & 0.795 & 14.225 & 13.945 \\
\hline 700.844 & 0.830 & 14.341 & 13.980 \\
\hline 700.865 & 0.860 & 14.298 & 14.004 \\
\hline 700.885 & 0.889 & 14.316 & 14.059 \\
\hline 700.915 & 0.930 & 14.128 & 13.934 \\
\hline 700.928 & 0.950 & 14.052 & 13.863 \\
\hline 700.937 & 0.962 & 13.955 & 13.823 \\
\hline 700.947 & 0.976 & 14.074 & 13.739 \\
\hline 700.957 & 0.990 & 13.955 & 13.843 \\
\hline 700.976 & 0.018 & 13.918 & 13.803 \\
\hline
\end{tabular}

at one of approximately the same two positions on the array during each night. After a sequence such that all the RR Lyraes in a given cluster were observed, sky frames were taken for the RR Lyraes by moving the telescope several hundred arcseconds away to a suitable location far from the cluster center. 
There are 118 observations of each M92 star and 60 of each M5 star.

\subsection{Data Analysis}

The IR array is slightly nonlinear. This was modeled on a global basis over the frame using a sequence of exposures of varying durations of a dome flat-field lamp. The linearity correction curve, whose maximum amplitude was about $4 \%$, is the ratio of the actual signal in a dome flat-field exposure $S_{A}$ of integration time $t_{A}$ divided by the signal expected by extrapolating from a 1 -s flat-field exposure $S_{1}$, $S_{A} /\left(S_{1} t_{A}\right)$. This function of observed signal $S_{A}$ was constant over the entire run. It was fit by a spline and the correction was applied to all pixels in each of the frames. Once the IR frames were linearized, the sky frame was subtracted from the object frame, and the difference frame was flattened. There were slight problems in flat fielding the data at $J$, and occasionally a coherent noise pattern (like microphonics) was seen in the flattened $J$ frames. This never exceeded $10 \mathrm{DN}$ in peak-to-peak amplitude.

Next the brightness of the object was measured using the aperture photometry code in Figaro. A 10 pixel diameter aperture, corresponding to $3.1 \mathrm{arcsec}$, was used. The variables M92-V6 and M5-V59 had adjacent reasonably bright (i.e., at lease 0.3 mag brighter than the variables at maximum light) neighbors on the same frame that could be used to define relative magnitudes, while M5-V8 had a somewhat fainter neighbor. The star 3.8-arcsec SW of M92-V6 was used to define the scale for that cluster, with $K=11.97 \mathrm{mag}$ and $J=12.54 \mathrm{mag}$. The star M5 III-9 in the numbering system of Arp (1962), 7.8-arcsec NE of M5V59, with $K=12.63 \mathrm{mag}$ and $J=13.20 \mathrm{mag}$, normalizes the M5 magnitudes. The remaining variables used the magnitude offsets from whichever of these two is in the relevant cluster.

This method of removing the influence of those timedependent effects such as extinction and seeing profiles was much more successful than just relying on the bright standard stars.

Standard Palomar airmass corrections were used. Note that the M5 observations spanned a more extreme range in airmass than did those of M92, reaching 1.9 on occasion before work on M5 was abandoned for the night.

\subsection{Crowding Corrections}

Only M92-V6 is sufficiently crowded for this to be a concern. There are two neighboring stars, the brighter one 3.8 arcsec to the SW, and a faint star 2.5 arcsec to the NW. The brighter star is about 1.9 mag brighter than the RR Lyrae M92-V6, while the fainter star is about $0.8 \mathrm{mag}$ fainter. The growth curve for magnitude as a function of aperture size was studied for the standard stars. The correction itself, and the range of variation of M92-V6, are both sufficiently small that use of a constant crowding correction seems suitable. As a result, a uniform crowding correction of $0.03 \mathrm{mag}$ was applied at $J$ and at $K$ to the observed photometry of M92-V6. The crowding correction is dominated by the contribution of the brighter, more distant, neighbor.

\subsection{Final Magnitudes}

The resulting $J$ and $K$ magnitudes, averaged over the pairs of frames, are given in Tables 7 and 8 for the M92 variables, and in Tables 9-12 for the M5 variables. Missing values occurred primarily for two reasons. Occasionally the filter mover software would malfunction, and the $J$ frames of a given measurement were in fact $K$ frames. In addition, toward the end of the second night, after M5 had already set, there was a period of rapidly fluctuating transparency. While the differential standard star on the frame of M92-V6 enabled us to retain full data coverage for that star, some $K$ measurements for M92-V1 were disregarded. Figures 3 and 4 show the light curves for the two M92 variables, and Fig. 5 is among the better of the M5 RR Lyrae light curves in that it has relatively complete phase coverage.

Although we expected, based on the observed count rates in the objects and in the sky, to achieve a photometric accuracy of $1 \%$ at $J$ and at $K$, the infrared light curves are rather ragged, and show that the accuracy achieved was significantly degraded, and is not better than $3 \%$ for each pair of measurements. Since the best light curves are those with local standards on the same frame, we believe that this is a consequence of possible small variations in atmospheric transmission, extinction deviations from the standard Palomar extinction law used, and variations in the stellar point spread function (PSF) affecting the fraction of total light included in our 3.1-arcsec aperture.

\section{APPLICATION OF THE MEAN MAGNITUDES}

As reviewed by Liu and Janes and by Carney et al. (1992), based on extensive Baade-Wesselink analyses of field RR Lyrae stars, these variables obey a number of well-established relationships. One of them is a relationship between absolute luminosity at $V$ and metallicity,

$$
\left\langle M_{V}\right\rangle=0.20( \pm 0.07)[\mathrm{Fe} / \mathrm{H}]+1.06( \pm 0.13) .
$$

We apply this without justification to the globularcluster RR Lyrae stars. We adopt reddening corrections of $E(B-V)=0.03$ for M5 (Zinn 1980) and 0.02 for M92 (Stetson and Harris 1988), with $A_{V}=3.1 E(B-V)$. For both clusters, the reddenings are small, so the exact choice from among the large number of published values is not very important. We adopt the metallicities of Zinn (1985) to obtain for the mean of the four M92 stars a distance modulus $(m-M)_{0}=14.48 \pm 0.04$, and for seven stars in M5 we obtain $(m-M)_{0}=14.25 \pm 0.05$.

Since we intend to use these light curves of RR Lyrae variables in M5 and M92 in a subsequent paper (Cohen 1992) on the Baade-Wesselink method, we defer detailed discussion here. Instead we emphasize a second possible method of distance determination. As discussed by Liu and 
TABLE 8

$J$ and $K$ Photometry of RR Lyrae M92-V6

\begin{tabular}{|c|c|c|c|}
\hline $\begin{array}{r}\text { Geocentric } \\
\text { Julian Date } \\
-2,447,000.0\end{array}$ & Phase & $\begin{array}{c}\mathrm{J} \\
(\mathrm{mag})\end{array}$ & $\begin{array}{c}\mathrm{K} \\
(\mathrm{mag})\end{array}$ \\
\hline 693.749 & 0.398 & 14.118 & 13.882 \\
\hline 693.792 & 0.471 & 14.161 & 13.897 \\
\hline 693.829 & 9.532 & 14.131 & 13.893 \\
\hline 693.864 & 0.590 & 14.217 & 13.936 \\
\hline 693.906 & 0.660 & 14.256 & 13.992 \\
\hline 693.918 & 0.680 & 14.257 & 13.998 \\
\hline 693.933 & 0.706 & 14.272 & 14.010 \\
\hline 693.946 & 0.726 & 14.318 & 14.041 \\
\hline 693.960 & 0.751 & 14.318 & 14.033 \\
\hline 693.974 & 0.774 & 14.316 & 14.061 \\
\hline 693.981 & 0.784 & 14.314 & 14.081 \\
\hline 694.663 & 0.922 & 14.259 & 13.917 \\
\hline 694.692 & 0.969 & 14.021 & 13.706 \\
\hline 694.721 & 0.018 & 13.925 & 13.793 \\
\hline 694.757 & 0.078 & 13.959 & 13.829 \\
\hline 694.794 & 0.141 & 14.009 & 13.829 \\
\hline 694.844 & 0.224 & 14.034 & 13.811 \\
\hline 694.877 & 0.278 & 14.047 & 13.892 \\
\hline 694.889 & 0.298 & 14.065 & 13.831 \\
\hline 694.901 & 0.318 & 14.099 & 13.860 \\
\hline 694.913 & 0.338 & 14.119 & 13.849 \\
\hline 694.923 & 0.355 & 14.108 & 13.845 \\
\hline 694.947 & 0.395 & 14.135 & 13.909 \\
\hline 694.960 & 0.416 & 14.145 & 13.869 \\
\hline 694.970 & 0.434 & 14.123 & 13.868 \\
\hline 694.982 & 0.453 & 14.187 & 13.885 \\
\hline 694.992 & 0.471 & 14.227 & 13.894 \\
\hline 698.684 & 0.623 & 14.285 & 13.952 \\
\hline 698.719 & 0.682 & 14.270 & 14.031 \\
\hline 698.763 & 0.754 & 14.339 & 14.020 \\
\hline 698.792 & 0.804 & 14.337 & 14.068 \\
\hline 698.898 & 0.980 & 13.988 & 13.873 \\
\hline 689.915 & 0.009 & 13.958 & 13.782 \\
\hline 698.943 & 0.055 & 13.980 & 13.849 \\
\hline 698.954 & 0.074 & 14.004 & 13.899 \\
\hline 698.976 & 0.111 & 14.038 & 13.821 \\
\hline 698.985 & 0.126 & 14.042 & 13.835 \\
\hline 699.685 & 0.291 & 14.024 & 13.827 \\
\hline 699.714 & 0.340 & 14.073 & 13.804 \\
\hline 699.742 & 0.386 & 14.142 & 13.854 \\
\hline 699.769 & 0.432 & 14.156 & 13.781 \\
\hline 699.812 & 0.504 & 14.246 & 13.828 \\
\hline 699.888 & 0.629 & & 13.973 \\
\hline 699.897 & 0.644 & 14.303 & 13.968 \\
\hline 699.915 & 0.674 & 14.302 & 14.003 \\
\hline 699.961 & 0.752 & 14.296 & 14.071 \\
\hline 699.982 & 0.787 & 14.288 & 14.110 \\
\hline 700.688 & 0.964 & 14.083 & 13.881 \\
\hline 700.717 & 0.012 & 13.975 & 13.822 \\
\hline 700.755 & 0.075 & 14.002 & 13.838 \\
\hline 700.787 & 0.128 & 14.049 & 13.759 \\
\hline 700.822 & 0.187 & 14.086 & 13.828 \\
\hline 700.847 & 0.229 & 14.066 & 13.789 \\
\hline 700.868 & 0.263 & 14.088 & 13.855 \\
\hline 700.889 & 0.298 & 14.089 & 13.861 \\
\hline 700.919 & 0.348 & 14.137 & 13.810 \\
\hline 700.931 & 0.369 & 14.146 & 13.810 \\
\hline 700.940 & 0.384 & 14.160 & 13.888 \\
\hline 700.951 & 0.401 & 14.183 & 13.879 \\
\hline 700.960 & 0.417 & 14.203 & 13.892 \\
\hline 700.979 & 0.449 & 14.221 & 13.862 \\
\hline
\end{tabular}

TABLE 9

$J$ and $K$ Photometry of RR Lyrae M5-V32

\begin{tabular}{|c|c|c|c|}
\hline $\begin{array}{r}\text { Geocentric } \\
\text { Julian Date } \\
-2,447,000.0\end{array}$ & Phase & $\begin{array}{c}\mathbf{J} \\
(\mathrm{mag})\end{array}$ & $\underset{(\mathrm{mag})}{\mathrm{K}}$ \\
\hline 693.681 & 0.583 & 14.472 & 14.185 \\
\hline 693.761 & 0.759 & 14.475 & 14.171 \\
\hline 693.806 & 0.858 & 14.594 & 14.368 \\
\hline 693.842 & 0.937 & 14.450 & 14.348 \\
\hline 693.872 & 0.000 & 14.086 & 13.952 \\
\hline 694.669 & 0.742 & 14.480 & 14.164 \\
\hline 694.697 & 0.802 & 14.414 & 14.079 \\
\hline 694.731 & 0.877 & 14.696 & 14.363 \\
\hline 694.765 & 0.953 & 14.392 & 14.166 \\
\hline 694.803 & 0.036 & 14.078 & 13.999 \\
\hline 694.853 & 0.145 & & 13.860 \\
\hline 698.658 & 0.455 & 14.397 & 14.055 \\
\hline 698.690 & 0.525 & 14.401 & 14.193 \\
\hline 698.728 & 0.610 & 14.442 & 14.231 \\
\hline 698.769 & 0.699 & 14.516 & 14.152 \\
\hline 698.827 & 0.825 & 14.529 & 14.184 \\
\hline 699.659 & 0.643 & 14.451 & 14.145 \\
\hline 699.692 & 0.716 & 14.426 & 14.110 \\
\hline 699.721 & 0.778 & 14.437 & 14.223 \\
\hline 699.749 & 0.840 & 14.511 & 14.236 \\
\hline 699.784 & 0.916 & 14.429 & 14.357 \\
\hline 700.665 & 0.839 & 14.499 & 14.034 \\
\hline 700.696 & 0.908 & 14.506 & 14.105 \\
\hline 700.732 & 0.986 & 14.021 & 13.901 \\
\hline 700.763 & 0.053 & 14.114 & 13.946 \\
\hline 700.801 & 0.138 & 14.047 & 13.954 \\
\hline 700.830 & 0.200 & 14.112 & 13.951 \\
\hline 700.853 & 0.250 & 14.102 & 13.932 \\
\hline
\end{tabular}

TABLE 10

$J$ and $K$ Photometry of RR Lyrae M5-V8

\begin{tabular}{|c|c|c|c|}
\hline $\begin{array}{r}\text { Geocentric } \\
\text { Julian Date } \\
-2,447,000.0\end{array}$ & Phase & $\begin{array}{c}\mathrm{J} \\
(\mathrm{mag})\end{array}$ & $\underset{(\mathrm{mag})}{\mathrm{K}}$ \\
\hline 693.691 & 0.454 & 14.195 & 13.850 \\
\hline 693.765 & 0.590 & 14.206 & 13.903 \\
\hline 693.810 & 0.671 & 14.329 & 13.974 \\
\hline 693.846 & 0.737 & 14.256 & 14.035 \\
\hline 693.874 & 0.790 & 14.377 & 14.027 \\
\hline 694.674 & 0.254 & 14.071 & 13.913 \\
\hline 694.701 & 0.302 & 14.085 & 13.756 \\
\hline 694.735 & 0.365 & 14.130 & 13.759 \\
\hline 694.769 & 0.427 & 14.162 & 13.905 \\
\hline 694.807 & 0.497 & 14.165 & 13.834 \\
\hline 694.857 & 0.589 & & 13.853 \\
\hline 698.662 & 0.554 & 14.270 & 13.963 \\
\hline 698.692 & 0.610 & 14.254 & 13.994 \\
\hline 698.697 & 0.618 & 14.262 & 13.875 \\
\hline 698.699 & 0.623 & 14.201 & 13.984 \\
\hline 698.732 & 0.682 & 14.274 & 13.927 \\
\hline 698.773 & 0.757 & & 13.934 \\
\hline 698.831 & 0.863 & 14.204 & 14.081 \\
\hline 699.663 & 0.387 & 14.074 & 13.660 \\
\hline 699.697 & 0.448 & 14.090 & 13.695 \\
\hline 699.724 & 0.499 & 14.110 & 13.876 \\
\hline 699.752 & 0.550 & 14.141 & 13.850 \\
\hline 699.788 & 0.616 & 14.129 & 13.812 \\
\hline 700.668 & 0.227 & 14.045 & 13.720 \\
\hline 700.699 & 0.283 & 14.081 & 13.763 \\
\hline 700.736 & 0.352 & 14.044 & 13.757 \\
\hline 700.765 & 0.405 & 14.094 & 13.860 \\
\hline 700.806 & 0.479 & 14.126 & 13.839 \\
\hline 700.832 & 0.527 & 14.125 & 13.839 \\
\hline 700.855 & 0.569 & 14.159 & 13.845 \\
\hline
\end{tabular}


TABLE 11

$J$ and $K$ Photometry of RR Lyrae M5-V12

\begin{tabular}{|c|c|c|c|}
\hline $\begin{array}{r}\text { Geocentric } \\
\text { Julian Date } \\
-2,447,000.0\end{array}$ & Phase & $\begin{array}{c}\mathrm{J} \\
(\mathrm{mag})\end{array}$ & $\begin{array}{c}\mathrm{K} \\
(\mathrm{mag})\end{array}$ \\
\hline 693.716 & 0.246 & 14.161 & 13.998 \\
\hline 693.770 & 0.362 & 14.277 & 14.005 \\
\hline 693.814 & 0.456 & 14.292 & 14.101 \\
\hline 693.850 & 0.533 & 14.316 & 14.045 \\
\hline 693.876 & 0.589 & 14.484 & 14.179 \\
\hline 694.678 & 0.304 & 14.293 & 13.914 \\
\hline 694.704 & 0.359 & 14.339 & 13.953 \\
\hline 694.739 & 0.433 & 14.322 & 14.013 \\
\hline 694.773 & 0.506 & 14.371 & 14.123 \\
\hline 694.810 & 0.585 & 14.451 & 14.128 \\
\hline 694.861 & 0.695 & & 14.215 \\
\hline 698.667 & 0.833 & 14.534 & 14.214 \\
\hline 698.703 & 0.908 & 14.529 & 14.279 \\
\hline 698.735 & 0.977 & 13.950 & 13.888 \\
\hline 698.777 & 0.067 & 14.203 & 13.868 \\
\hline 698.836 & 0.193 & 14.221 & 13.939 \\
\hline 699.666 & 0.968 & 14.044 & 13.996 \\
\hline 699.699 & 0.039 & 14.025 & 13.920 \\
\hline 699.727 & 0.098 & 14.042 & 13.964 \\
\hline 699.756 & 0.159 & 14.109 & 13.851 \\
\hline 699.797 & 0.248 & 14.182 & 13.877 \\
\hline 700.672 & 0.118 & 14.083 & 13.852 \\
\hline 700.702 & 0.183 & 14.058 & 13.929 \\
\hline 700.739 & 0.262 & 14.130 & 13.938 \\
\hline 700.768 & 0.324 & 14.262 & 13.917 \\
\hline 700.808 & 0.410 & 14.243 & 13.972 \\
\hline 700.836 & 0.469 & 14.404 & 14.043 \\
\hline 700.856 & 0.513 & 14.350 & 14.026 \\
\hline
\end{tabular}

TABLE 12

$J$ and $K$ Photometry of RR Lyrae M5-V59

\begin{tabular}{|c|c|c|c|}
\hline $\begin{array}{r}\text { Geocentric } \\
\text { Julian Date } \\
-2,447,000.0\end{array}$ & Phase & $\begin{array}{c}\mathbf{J} \\
(\mathrm{mag})\end{array}$ & $\begin{array}{c}\mathrm{K} \\
(\mathrm{mag})\end{array}$ \\
\hline 693.725 & 0.463 & 13.932 & 13.617 \\
\hline 693.777 & 0.559 & 14.003 & 13.646 \\
\hline 693.817 & 0.634 & 14.003 & 13.687 \\
\hline 693.853 & 0.699 & 14.050 & 13.743 \\
\hline 693.880 & 0.749 & 14.127 & 13.794 \\
\hline 694.683 & 0.231 & 13.934 & 13.666 \\
\hline 694.708 & 0.276 & 13.892 & 13.651 \\
\hline 694.742 & 0.340 & 13.951 & 13.651 \\
\hline 694.778 & 0.406 & 13.966 & 13.695 \\
\hline 694.814 & 0.472 & 13.995 & 13.724 \\
\hline 694.865 & 0.566 & & 13.712 \\
\hline 698.672 & 0.589 & 14.019 & 13.705 \\
\hline 698.708 & 0.656 & 14.003 & 13.673 \\
\hline 698.740 & 0.715 & 14.023 & 13.693 \\
\hline 698.781 & 0.791 & 14.042 & 13.735 \\
\hline 698.840 & 0.901 & 14.083 & 13.791 \\
\hline 699.669 & 0.430 & 13.941 & 13.575 \\
\hline 699.702 & 0.491 & 13.941 & 13.571 \\
\hline 699.731 & 0.543 & 13.978 & 13.649 \\
\hline 699.758 & 0.594 & 14.002 & 13.596 \\
\hline 699.799 & 0.670 & 14.055 & 13.667 \\
\hline 700.674 & 0.284 & 13.912 & 13.544 \\
\hline 700.705 & 0.341 & 13.888 & 13.584 \\
\hline 700.743 & 0.411 & 13.926 & 13.571 \\
\hline 700.776 & 0.471 & 13.903 & 13.587 \\
\hline 700.812 & 0.538 & 14.036 & 13.665 \\
\hline 700.839 & 0.588 & 14.042 & 13.649 \\
\hline 700.858 & 0.624 & 13.992 & 13.690 \\
\hline
\end{tabular}

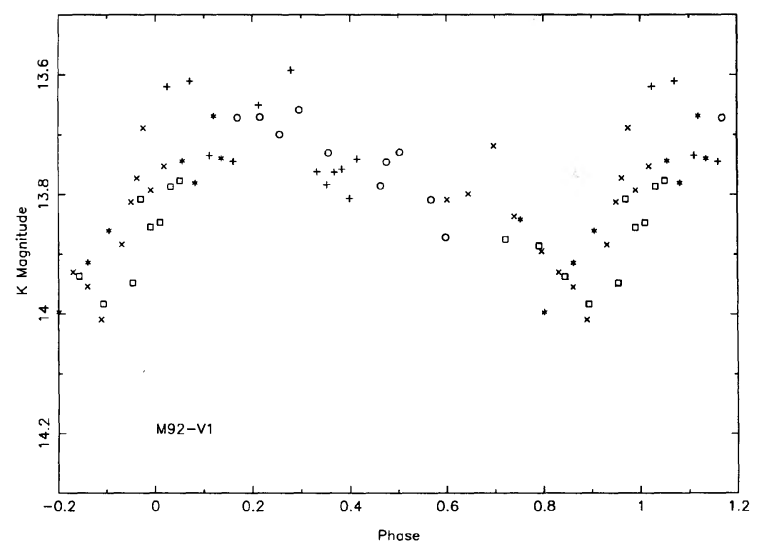

FIG. 3-The $K$ band light curve for the RR Lyrae variable M92-V1 is shown as a function of phase. The different symbols denote different nights of observing.

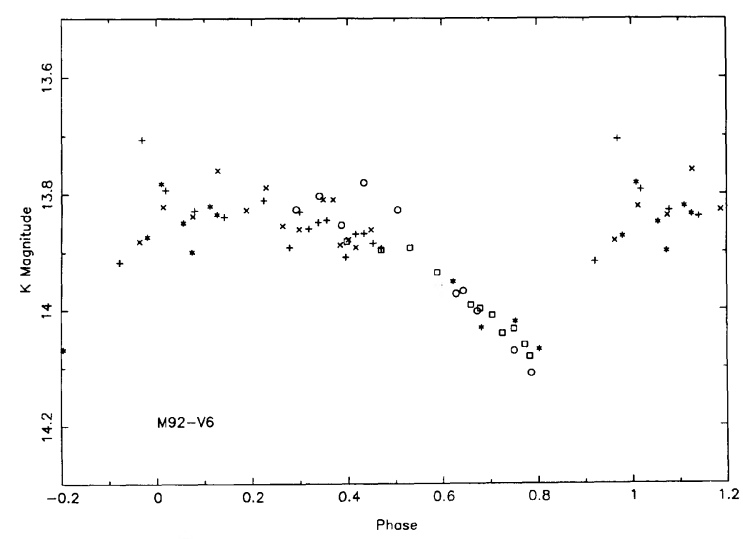

FIG. 4 The same as Fig. 3 for the RR Lyrae variable M92-V6.

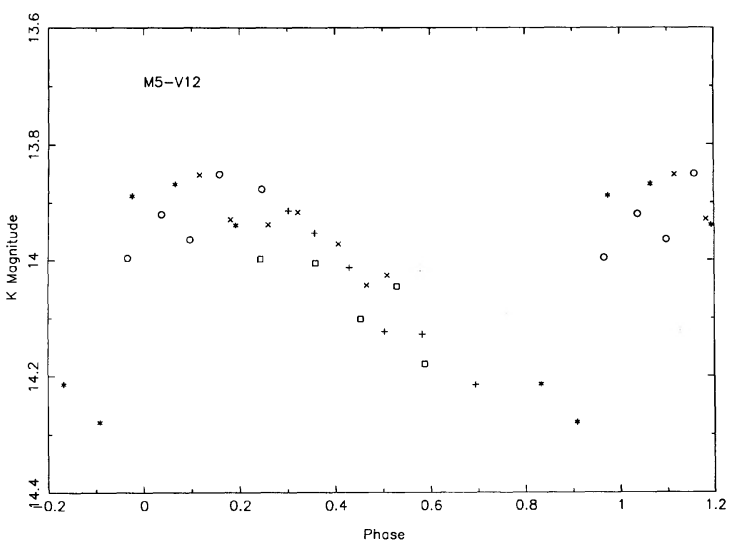

FIG. 5-The same as Fig. 3 for the RR Lyrae variable M5-V12. 
Janes (1991), Carney et al. (1992), and Longmore et al. (1990), among the field RR Lyrae variables there is also a relationship between absolute $K$ magnitude and period. It has the form (from Liu and Janes)

$$
\left\langle M_{K}\right\rangle=-2.44( \pm 0.24) \log (P)-0.90( \pm 0.14)
$$

The observed mean $K$ magnitudes were calculated from the $K$ measurements from a smooth hand drawn light curve drawn through the $K$ light curve which was digitized every 0.02 interval in phase. For M5-V32, where there were no measurements over a a significant phase interval, the $K$ light curve of M92-V1 was used, appropriately scaled to the amplitude of the variation at $K$, to interpolate over the missing region. Extinction corrections at $K$ of $0.11 A_{V}$, where $A_{V}=3.1 E(B-V)$, have been applied in both clusters. Applying the extinction corrected mean $K$ magnitudes for the six variables from Table 13 yields a distance modulus to M5 of $14.10 \mathrm{mag}( \pm 0.11$ ) (which goes up to 14.15 if M5-V59, which seems excessively bright, is omitted) and to M92 of $14.30 \mathrm{mag}( \pm 0.04)$. These values are 0.16 \pm 0.02 mag brighter than were obtained from the visual light curves. This will be discussed further in Cohen (1992).

We close with a comparison with Storm et al. For M92-V1 the agreement in $\langle K\rangle$ is very good, 13.82 versus our value of 13.80 . For M5-V8 the situation is poor, and the $\langle K\rangle$ are 13.99 versus our value of 13.86 . The shape of the light curve looks the same, so there may be a problem with the M5 magnitude zero point. The cause of this discrepancy is not understood.

J. G. C. is grateful to Gaston Araya Machining and to the Caltech Recycling Center for financial support. K. M. is supported by NSF Grant No. AST-8920897. We are grateful to D. Carico for help with the observing on several nights. Ben Holland helped with the observing at the 60 -in. telescope.

\section{REFERENCES}

Arp, H. C. 1962, ApJ, 135, 311

Buonanno, R., Buscema, G., Corsi, C. E., Iannicola, G., Smgriglio, F., and Fusi Pecci, F. 1983, A\&AS, 53, 1

Buonanno, R., Corsi, C. E., and Fusi Pecci, F. 1981, MNRAS, 196,435

Carney, B. W., Storm, J., and Jones, R. V. 1992, ApJ, 386, 663

Carney, B. W., Storm, J., Tramell, S., and Jones, R. V. 1992, PASP, 104, 56

Cohen, J. G. 1992, ApJ, in press

Cohen, J. G. and Gordon, G. A. 1987, ApJ, 247, 869

Coutts, C. M., and Sawyer Hogg, H. 1969, Publ. David Dunlop Obs., 3, 1

Elias, J. H., Frogel, J. A., Matthews, K., and Neugebauer, G. 1982, AJ, 87, 1029

Jameson, R. F., Fernley, J. A., and Longmore, A. J. 1987, in Stellar Pulsations, ed. A. N. Cox and W. M. Starrfield, Lecture Notes in Physics, 274, 239

Jones, R. V., Carney, B. W., and Latham, D. W. 1988, ApJ, 332, 206

Kukharkin, B. V., and Kukharkina, N. P. 1980, Variable Stars, 21,365

Liu, T. X., and Janes, K. A. 1991, The Formation and Evolution of Star Clusters, ed. K. Janes, p. 278

Longmore, A. J., Dixon, R., Skillen, I., Jameson, R. F., and Fernley, J. A. 1990, MNRAS, 247, 684

Richer, H. B., and Fahlman, G. 1987, ApJ, 316, 189

Sandage, A. R. 1990, ApJ, 350, 631

Sandage, A. R., and Walker, M. F. 1966, ApJ, 143, 313

Sawyer Hogg, H. 1973, Publ. David Dunlap Obs., 3, 1

Stetson, P. B. 1987, PASP, 99, 191

Stetson, P. B., and Harris, W. E. 1988, AJ, 96, 909

Storm, J., Carney, B. W., and Beck, J. A. 1991, PASP, 103, 1264

Thuan, T. X., and Gunn, J. E. 1976, PASP, 88, 543

Zinn, R. J. 1980, ApJS, 42, 19

Zinn, R. J. 1985, ApJ, 293, 424 